\title{
Benefits of Mechanical Manipulation of the Sacroiliac Joint: A Transient Synovitis Case Study
}

\author{
Mitchell Goldflies ${ }^{1}$, Michael Rosen ${ }^{2}$ and Brady Hauser ${ }^{3 *}$ \\ ${ }^{1}$ Orthopedic Surgeon, USA \\ ${ }^{2}$ Doctor of Chiropractic Medicine, USA \\ ${ }^{3}$ St. Georges University, USA \\ *Corresponding author: Brady Hauser, St. Georges University, School of Medicine, USA
}

Submission: 海 April 11, 2018; Published: 眥 May 15, 2018

\begin{abstract}
Transient synovitis is a diagnosis of exclusion for acute hip pain in children. It has largely been associated with viral and traumatic etiologies despite most of the cases being idiopathic in nature. The purpose of this study is to present a patient with transient synovitis that also demonstrates Sacroiliac Joint dysfunction. Established Sacroiliac Joint (SIJ) manipulation technique was used to resolve his symptoms. The patient in this study displayed physical and radiological finding consistent with transient synovitis of the hip associated with SIJ dysfunction. We theorize that mechanical joint dysfunction of the SIJ may lead to changes in the tension-length relationship of the iliopsoas muscle predisposing the hip joint to tendinitis and bursitis. This results in the clinical presentation of transient synovitis of the hip. Our patient was successfully treated with SIJ manipulation. This study emphasizes the importance of a thorough spinal examination in patients presenting with transient synovitis to rule out a mechanical pathology. Our case illustrates the efficacy of spinal manipulation in treating SIJ dysfunction. We propose that this evaluation and treatment option should be considered with patients diagnosed with transient synovitis of the hip.
\end{abstract}

Keywords: Transient synovitis; Toxic synovitis; Hip pain; Sacroiliac joint; Mechanical joint dysfunction; Spinal manipulation

\section{Introduction}

Hip pain in a young child or adolescent has a wide differential diagnosis that can be narrowed by the patient's age, presentation, radiological findings, and lab results. Transient synovitis is largely

a diagnosis of exclusion [1,2]; it is imperative to rule out other diseases associated with pediatric hip pain (such as: Legg-CalvePerthe Disease, Slipped Capital Femoral Epiphysis, and Septic Hip) prior to diagnosing transient synovitis.

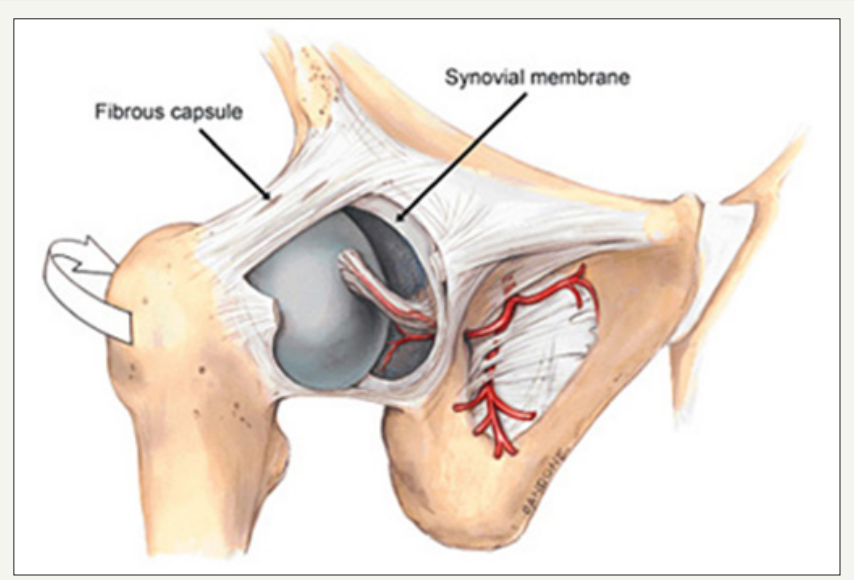

Figure 1: Artist interpretation of the synovial membrane found deep to the fibrous capsule. The fibrous capsule surrounds the hip and attaches to the femoral neck.

Source: (Image recovered from: Coronaphysio.net; Corona Physical Therapy, 1113 S Main St. Corona, CA 92882). 
Transient synovitis (also called toxic synovitis) is the most common cause of acute hip pain and limp in young children [1,3]; it affects boys more than girls [3,4], and is usually self-limited. It is defined by inflammation of the inner lining of the hip joint capsule (Figure 1). Occurrence is usually between the age of three and ten years old [5] and the exact cause is unknown. Traditionally, diagnosis is made via clinical finding of hip pain and limp in a young child with a history viral upper respiratory infection (URI) or recent trauma. The patient will present with limited range of motion of the affected hip joint and pain on ambulation. Blood test may demonstrate inflammation (elevations in ESR and CRP); an ultrasound (U/S) or Magnetic Resonance Imaging (MRI) of the hip may show effusion. Treatment is with a non-steroidal antiinflammatory drug (NSAID) and limited weight bearing activities. symptom usually subsides in seven to ten days (Moses, 2007). Some populations may continue to have symptoms for several weeks. There is a recurrence rate of $4-17 \%$ [6].

Transient synovitis has widely been associated with URI and/ or recent trauma despite only $30 \%$ and $5 \%$, respectively, of cases report those specific etiologies [4]. Other etiologies such as an underlying mechanical musculoskeletal pathology have not been adequately explored. This case examines a five-year-old male with Sacroiliac joint dysfunction who was diagnosed with Transient
Synovitis and successfully treated with manipulation of the SIJ.

\section{Case Report}

A five-year-old male with no significant past medical history presented to the Emergency Department (ED) with left hip pain and limited range of motion. The patient was alert, oriented, and in obvious discomfort. All vital signs were within normal limits. The patient's mother denied any trauma or recent illnesses. She also denies generalized symptoms such as fever, nausea, and vomiting. The pain was exacerbated by weight bearing activities, including walking and standing. The pain was reduced by restricting weight bearing on the left lower extremity and holding the left hip in a flexed and externally rotated position.

Gait evaluation demonstrated an antalgic gait on the left side. Physical examination demonstrated diffusely soft tender tissues about the left hip upon palpation. Limited active and passive range of motion (ROM) of the left hip was apparent due to pain. Visual inspection indicated that the left leg was shorter supine than the right leg. Boney landmarks (Anterior Superior Iliac Spine, Iliac Crest, and Posterior Superior Iliac Spine) of the pelvis were asymmetrical. Neuro-vascular examination was normal bilaterally in the lower extremities. Radiographs (AP and frog-leg lateral pelvis) demonstrated a pelvic tilt (Figure 2) and were otherwise normal.

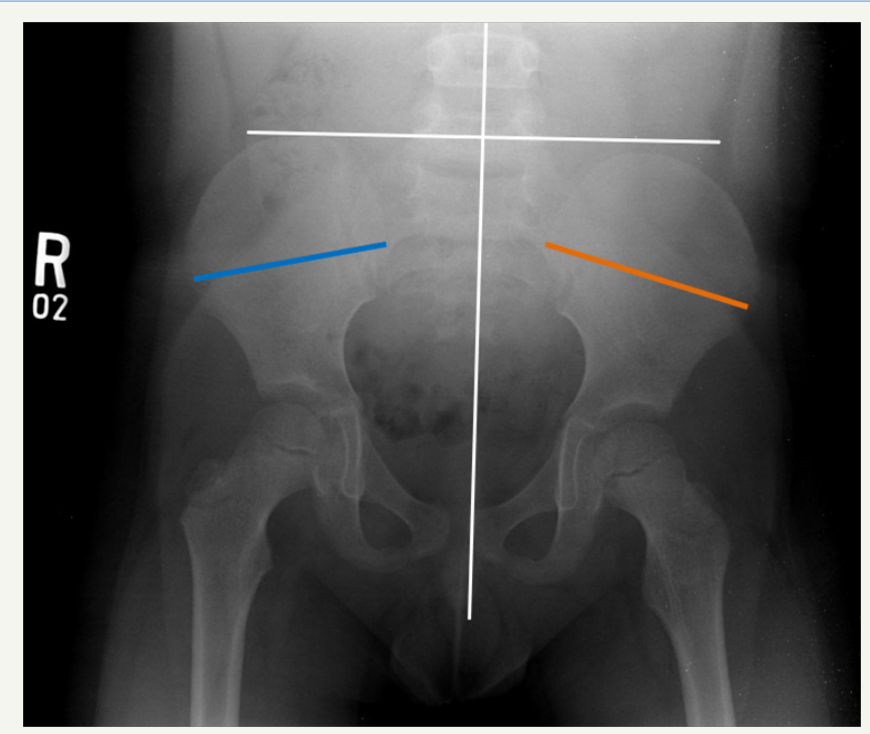

Figure 2: AP view of pelvis. A normal plum line (white vertical line) should run through the spinous processes all the way down to the center of pubic symphysis. In this case, plum line is not aligned with the center of pubic symphysis. Furthermore, the line perpendicular to the plum line (horizontal white line) does not line up the superior borders of the pelvic brim as it should. Also, the lines spanning the iliac crest are not of the same length with the orange line longer than the blue line. All of these suggest a pelvic malrotation in which the left side of the sacrum has undergone a unilateral flexion causing the leg length discrepancy.

Laboratory work-up included basic metabolic panel (BMP), erythrocyte sedimentation rate (ESR), urinalysis(UA), and complete blood count (CBC). All results were within normal limits. The patient was clinically non-toxic, and a febrile. These findings made the probability of septic arthritis less than 0.2\%3 (WBC: 10000 cells/microL; ESR: $4 \mathrm{~mm} / \mathrm{hr}$ ). The patient remained in moderate distress and was given an IV morphine drip, which did not alleviate his pain. An Orthopedic medicine specialist was consulted and determined the patient's hip pain may be related to an iliopsoas tendinitis because of the sacroiliac joint dysfunction. He noted an anterior tilt of the sacrum. After discussing the procedure, risks, benefits and alternatives to spinal manipulation the patient's mother gave consent to proceed. 
The patient's sacroiliac joints, L4-L5, and L5-S1 facet joints were manipulated while the patient was positioned prone. Following the manipulation, it was noted that the patient's leg length discrepancy and left hip pain resolved, ROM improved, and the patient was able bear weight on his left lower extremity with minimal discomfort. The patient was prescribed Children's strength ibuprofen and restricted activity upon discharge. Two days following evaluation and treatment in the ED the patient returned for follow-up at the Orthopedic Medicine outpatient clinic. The patient's mother stated that the patient's complaints resolved within 24 hours post SIJ manipulation and he had returned to normal activity and was currently asymptomatic. The patient stated that none of the prescribed NSAID's were taken. On physical exam, the patient's gait evaluation was normal, left hip joint was non-tender with full active and passive ROM without pain and the leg lengths supine were equal.

\section{Discussion}

Transient synovitis of the hip is one of the most common causes of non-traumatic hip pain in children presenting with unilateral hip pain and limited range of motion. Children with transient synovitis usually have an antalgic gait and prefer to keep the affected hip abducted and externally rotated [7]. Fever is usually absent, and children present clinically non-toxic. Patients who are febrile and have other elevated inflammatory markers should be evaluated for septic hip arthritis and undergo arthrocentesis.

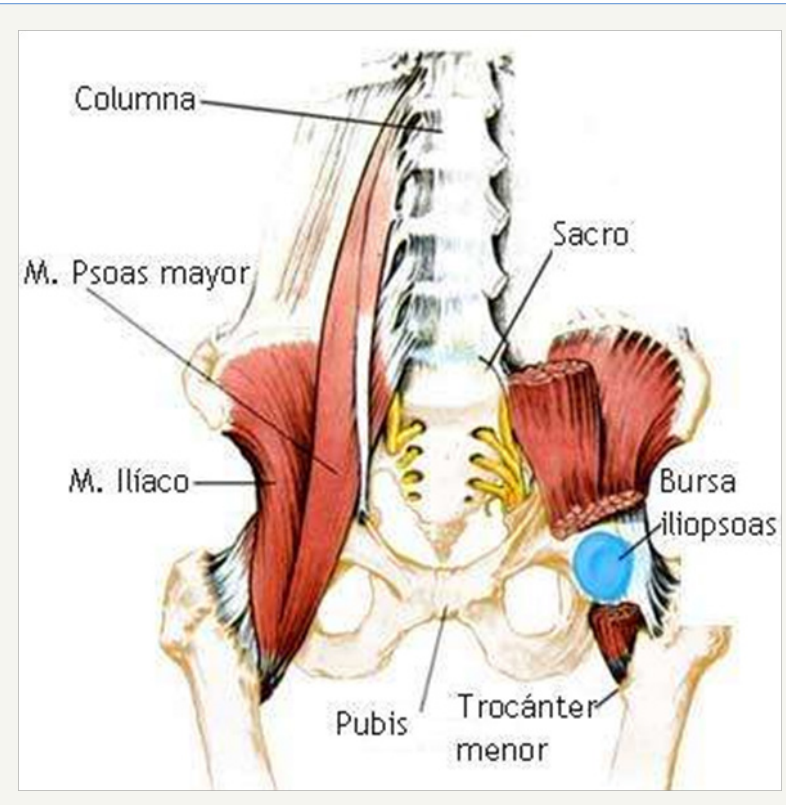

Figure 3: Artist interpretations of origin and insertion of the Psoas Major, Iliacus, and the location of the Iliopsoas Bursa. This image demonstrates the relationship between the hip flexor muscles and underlying bone structure.

Source: (Image recovered from: https://www.cabrillo.edu/ rnolthenius/med/AnteriorHipPain/index.html).

Patients with transient synovitis of the hip present with acute hip pain upon standing and/or ambulation. They will have an associated limp on the affected side. They may be a febrile, demonstrate leukocytosis and with elevated inflammatory markers. Hip synovitis is an inflammatory process of the synovial lining of the hip joint. Robbins et al, used Ultrasound (U/S) to compare healthy children with those diagnosed with transient synovitis. They demonstrated that joint capsule thickening was related to an effusion and not an infectious process [8]. If the effusion had been caused by an infectious process imaging would have shown synovial hypertrophy due to synovial cell inflammation and the effusion would be secondary to the infected synovial membrane. There was no evidence of synovial hypertrophy; which contradicts a viral etiology. A possible explanation is that the effusion originated in nearby structures rather than the synovial membrane itself. The Iliopsoas tendon or bursa could be the source of the effusion within the joint capsule in transient synovitis. The hip capsule surrounds the femoral neck (Figure 1). The iliopsoas, a combination of the iliacus and psoas muscle, passes anterior to the hip capsule in a groove between the anterior inferior iliac spine laterally and the iliopectineal eminence medially. The iliopsoas inserts at the lesser trochanter of the femur (Figure 3). The external surface of the anterior hip capsule is separated from the iliopsoas musculotendinous junction by the iliopectineal bursa [9]. From cadaver studies, $15 \%$ of iliopectineal bursae communicates with the joint capsule [10]. Due to the proximity, tendinitis of the iliopsoas tendon and inflammation of the iliopsoas bursa may be the etiology of the hip joint effusion.

During childhood growth spurts hip flexor muscles may not elongate to accommodate with the lengthening of the proximal femur. This results in relative shortening of the hip flexor muscles and overlying fascia. The tightness of the iliopsoas, tensor fascia lata, and/or rectus femoris can lead to inhibition of the gluteus maximus resulting in an anterior tilt of the sacroiliac joint [11]. 
The anterior tilt, as noted on this patient, increases the likelihood of repetitive compression between the iliopsoas tendon and the anterior capusulo-labral complex. The iliopsoas tendon normally loses contact with the femoral capsule at 14 degrees of flexion. The anterior tilt of pelvis causes the Anterior Superior Iliac Spine (ASIS) and pubic symphysis to shift relative position keeping the hip joint capsule in contact with the iliopsoas tendon through more hip flexion than normal [12]. This would suggest that the inflammation of the iliopsoas tendon and bursa are due to friction. This inflammation and resulting inflammatory fluid may diffuse into the hip capsule resulting in an acute hip effusion. Through SIJ manipulation we corrected the pelvic dysfunction in this patient. This treatment resolved the acute symptoms hip joint symptoms as well as the chronic somatic dysfunction etiology of our patient's transient synovitis. The manipulation reduced the amount and length of contact and friction between the iliopsoas tendon and the anterior capusulo-labral hip joint complex. The manipulation resolved the acute hip symptoms and potentially reduced the incidence of recurrence.

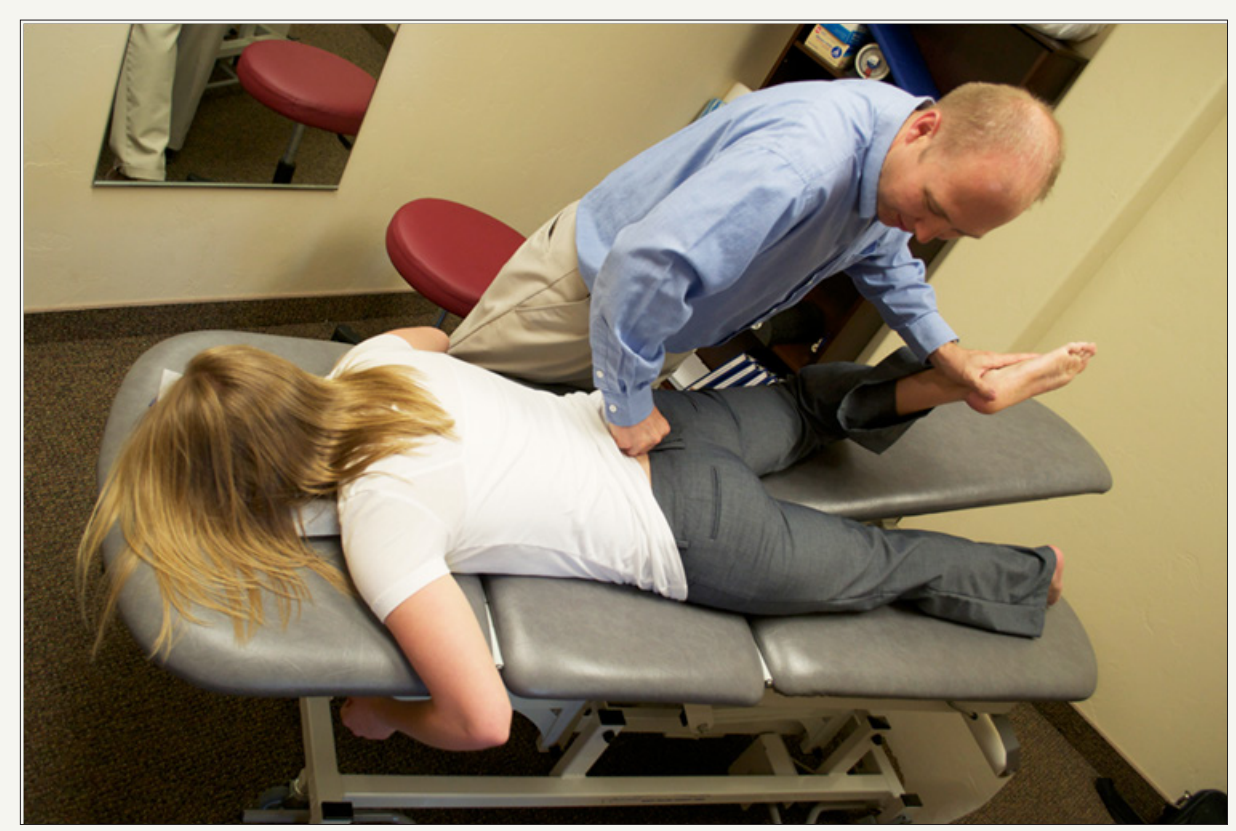

Figure 4: While the patient is prone, physician applying anterior and superior pressure on the pelvic apex while holding the affected hip in extension. The patient is instructed to take a deep breath and hold throughout this process.

Lumbo-pelvic dysfunction indicates that the physiological movements of sacrum on the ilium are reduced. This is often due to an imbalance of tension and tone of the muscles and ligaments that span the hip joint. In this case, a unilateral anterior sacral nutation (also called sacral flexion) was noted while the sacral base was anteriorly tilted on one side. This resulted in hypertonus of the piriformis and iliopsoas muscles (hip flexors) and a unilateral tilting of the pelvis that was demonstrated by a functional leg length difference. The treating physician addressed this issue by maintaining pressure on the inferior lateral aspect of sacral apex while the patient is in a prone position; manually unlocking the sacroiliac joint back to its physiologic position [13] (Figure 4). This procedure should stretch the iliopsoas tendon while forcing it to lose contact with hip capsule and alleviating the acute symptoms while addressing the chronic abnormal alignment.

This distortion of the normal tension-length relationship in two joint muscles, related to lumbo-pelvic dysfunction and leg length discrepancy, appears to also play a role in other pediatric musculoskeletal conditions. These conditions include unilateral knee tendinitis, Patello- Femoral Compression Syndrome, Osgood
Schlatter disease, Sinding-Larsen-Johansson syndrome, Iliac Apophysitis, and Sever's disease. This case demonstrates the efficacy of spinal manipulative therapy in treating lumbo-pelvic dysfunction; therefore, mechanical manipulation may be a potential treatment for the aforementioned conditions.

\section{Conclusion}

A five-year-old male was diagnosed with transient synovitis unrelated to an infectious or a traumatic etiology. Complete spinal physical examination and radiographs of the lumbar spine and pelvis demonstrated a lumbo-pelvic dysfunction. It is believed that dysfunction of the SIJ lead to iliopsoas tendonitis which progressed into a transient synovitis of the hip joint. The patient's SIJ dysfunction was successfully treated with SIJ manipulation therapy. Patients who have a suspected transient synovitis of the hip joint should be evaluated mechanically for lumbo-pelvic dysfunction and leg length discrepancy. Following initial treatment continuation of soft tissue rebalancing, core strengthening, and proprioception training is also required to allow the condition to completely resolve and reduce the chance of recurrence. Sacroiliac dysfunction may be 
an underlying etiology for transient synovitis of the hip joint due to the alteration the pelvic anatomy and associated distortion of the tension-length relationship of the muscles that span the sacroiliac and hip joints.

Further investigation and a larger study are needed to explore the prevalence of SIJ dysfunction in pediatric patients diagnosed with transient synovitis of the hip and knee. Further studies exploring the efficacy between pediatric patients presenting with transient synovitis due SIJ dysfunction and spinal manipulation are necessary to support spinal manipulation as a viable treatment option for these conditions.

\section{References}

1. Hart JJ (1996) Transient synovitis of the hip in children. Am Fam Physician 54(5): 1587-1591.

2. Tolat V, Carty H, Klenerman L, Hart CA (1993) Evidence for a viral aetiology of transient synovitis of the hip. J Bone Joint Surg Br 75(6): 973-974.

3. Scott Moses (2007) Transient hip tenosynovitis. Family Practice notebook.

4. Vijbrief AS, Bruijnzeels MA, Van Der Wouden JC, Van Suijlekom-Smit LW (1992) Incidence and management of transient synovitis of the hip: a study in dutch general practice. Br J Gen Pract 42(363): 426-468.

5. Quintos-Macasa AM, Serebro L, Menon Y (2006) Transient synovitis of the hip in an adult. South Med J 99(2): 184-185.

6. Mattick A, Turner A, Ferguson J, Beattie T, Sharp J (1999) Seven year follow up of children presenting to the accident and emergency department with irritable hip. J Accid Emerg Med 16(5): 345-347.

7. Fischer SU, Beattie TF (1999) The limping child: epidemiology, assessment and outcome. J Bone Joint Surg Br 81(6): 1029-1034.

8. Robben SG, Lequin MH, Diepstraten AF, Den hollander JC, Entius CA, et al. (1999) Anterior joint capsule of the normal hip and in children with transient synovitis: US study with anatomic and histologic correlation. Radiology 210(2): 499-507.

9. Johnston CA, Wiley JP, Lindsay DM, Wiseman DA (1998) Iliopsoas bursitis and tendinitis. A review. Sports Med 25(4): 271-283.

10. Van riet YE, Van vroonhoven TJ, Van der werken C, Berkhoudt AN (1996) Bursae communicating with the hip joint. A report on 2 cases. Acta Orthop Belg 62(2): 120-122.

11. Khan K, Cook JL, Maffulli N (2000) Tendinopathy in the active person: Separating fact from fiction to improve clinical management. Am J Med Sports 2(2): 89-99.

12. Domb BG, Shindle MK, Mcarthur B, Voos JE, Magennis EM, et al. (2011) Iliopsoas impingement: a newly identified cause of labral pathology in the hip. HSS J 7(2): 145-150.

13. Isaacs ER, Bookhout MR, Bourdillon JF (2002) Bourdillon's spinal manipulation. Butterworth-Heinemann Medical, UK.

14. Haueisen DC, Weiner DS, Weiner SD (1986) The characterization of "transient synovitis of the hip" in children. J Pediatr Orthop 6(1): 11-17.

15. Kocher MS, Zurakowski D, Kasser JR (1999) Differentiating between septic arthritis and transient synovitis of the hip in children: an evidence-based clinical prediction algorithm. J Bone Joint Surg Am 81(12): 1662-1670.

16. Gilroy AM, MacPherson BR, Ross LM (2012) Atlas of Anatomy. Thieme Medical Publishers, Germany.
Creative Commons Attribution 4.0 International License

For possible submissions Click Here

\section{Submit Article}

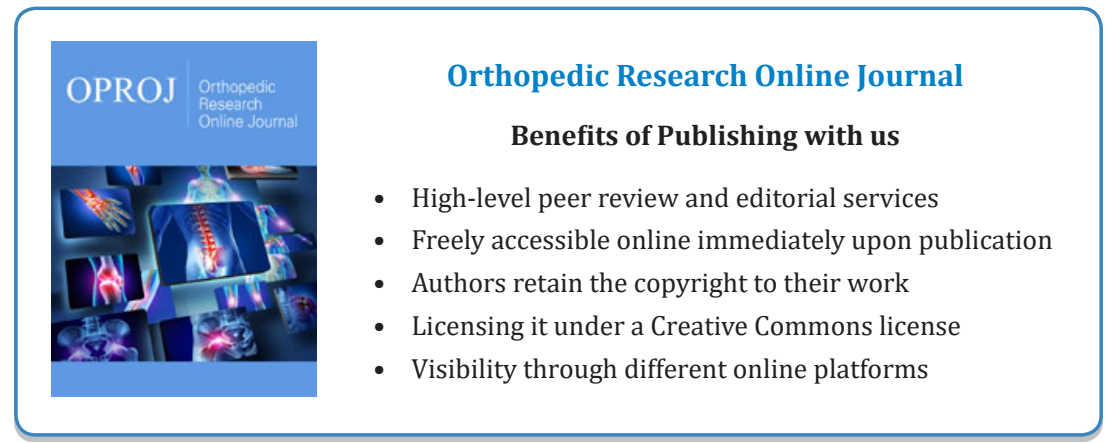

